Vaccinating to protect others: The role of self-persuasion and empathy among young adults

Dariusz Drążkowski ${ }^{1}$, Radosław Trepanowski ${ }^{1}$, and Valerie Fointiat ${ }^{2}$

${ }^{1}$ Faculty of Psychology and Cognitive Science, Adam Mickiewicz University, Poznan, Wielkopolska, Poland

2

UFR Arts, Lettres, Langues et Sciences Humaines, Aix-Marseille Université, Marseille, France

\begin{abstract}
Author Note
The dataset is available for free download from the Open Science Framework (https://osf.io/ckf2v/?view only=42799b0d579b437c8cb926ccfddbfba0).
\end{abstract}

The research has been funded from the competition entitled "Research on COVID-19" from the funds of Adam Mickiewicz University in Poznan (agreement no. 22/2020).

The authors declare that there is no conflict of interest.

Both studies were carried out with the guidelines of the Declaration of Helsinki and all its amendments and the guidelines of the Faculty of Psychology and Cognitive Science Ethics Committee at Adam Mickiewicz University in Poznan. All participants provided informed consent.

Correspondence concerning this article should be addressed to Dariusz Drążkowski, Faculty of Psychology and Cognitive Science, Adam Mickiewicz University, 89 Szamarzewskiego Street, PL-60-568, Poznan, Poland, E-mail: dardra@amu.edu.pl 
Vaccinating to protect others: The role of self-persuasion and empathy among young adults

\begin{abstract}
Direct persuasion (providing arguments) is usually a less effective influence technique than self-persuasion (self-creation of arguments). As most young adults are unafraid to contract COVID-19, self-persuasion focused on protecting the health of others may be an effective method of increasing vaccination rates in this population. Thus, empathy seems to be a crucial factor affecting vaccination intentions. We conducted two experiments using the theory of planned behavior. Study $1(\mathrm{~N}=366)$ compared the effectiveness of self-persuasion targeted at others' health versus personal health and direct persuasion in encouraging COVID-19 vaccination intention. Results indicate that among young adults, self-persuasion targeted at others' health (compared to other forms of persuasion) has an indirect effect on vaccination intention through utility and social norms beliefs. Moreover, as empathy increases, utility, social norms, and control beliefs increase, which is associated with a greater COVID-19 vaccination intention. Study $2(\mathrm{~N}=375)$ investigated the applicability of self-persuasion in health communication through a poster framed as an open-ended question. We found that the poster with self-persuasion targeted at others' health, enhanced vaccination intention, compared with a direct persuasion poster. Together, our findings demonstrate the potential of self-persuasion targeted at others' health in increasing COVID-19 vaccination uptake among young adults.
\end{abstract}

Keywords: COVID-19 vaccination, health communication, self-persuasion, planned behavior theory, empathy 


\section{Vaccinating to protect others: The role of self-persuasion and empathy among young}

\section{adults}

Vaccination against COVID-19 is a solution to overcome the ongoing pandemic (Yamey et al., 2020). While several vaccines have been developed, the effectiveness of vaccination programs depends on high uptake rates among the population. In particular, young people do not want to be vaccinated against COVID-19 as they are less afraid of contracting the disease (Lazarus et al., 2020; Robertson et al., 2021). Thus, it is crucial to develop evidence-based health communication and encourage young adults to vaccinate against COVID-19. Approaches that examine persuasive techniques in tandem with motivational factors can address these challenges. In this study, we investigate the effectiveness of self-persuasion targeted at protecting the health of others to encourage young adults to vaccinate against COVID-19.

\section{Self-persuasion}

Different governments have been using various strategies to promote vaccine uptake, for instance, by using persuasive communications (e.g., Rosen et al., 2021). Some studies have demonstrated that direct persuasion could increase COVID-19 vaccination intention (Ashworth et al., 2021). However, other studies have shown that attempts to encourage COVID-19 vaccination through direct persuasive messages are ineffective (e.g., Kachurka et al., 2021). Direct persuasion may also lead to a boomerang effect as it tends to trigger the message recipients' psychological reactance (Grandpre et al., 2003). Opposite effects of direct persuasion were observed in prior studies when people were encouraged to vaccinate (e.g., Nyhan et al., 2014).

Compared with direct persuasion, a subtler persuasive technique is self-persuasion, the process of generating one's own arguments towards a specific issue (Aronson, 1999). 
Creating one's own argument in opposition to receiving arguments from external sources (as in direct persuasion) reduces the risk of psychological reactance (Mussweiler \& Neumann, 2000; Wilson \& Brekke, 1994). Individuals creating arguments that contradict their attitudes experience cognitive dissonance, which motivates them to seek consistency in their cognition by realigning their attitudes to match the created arguments (Festinger, 1957). Baldwin et al. (2013) found another mechanism underlying effective self-persuasion. The content of a self-generated argument is tailored to people's concerns to a greater extent than arguments from external sources. Therefore, people think more positively about and evaluate their arguments more favorably than external arguments. A considerable body of research has shown that self-persuasion leads to improvements in health-related behaviors. For instance, Baldwin et al. (2017) demonstrated that self-persuasion might increase parents' intention to vaccinate their adolescent child against Human Papillomavirus (HPV). Moreover, Drążkowski et al. (2020) revealed that self-persuasion significantly impacted the moral obligation to socially isolate during the COVID-19 pandemic, and through it on self-isolation intention.

\section{Motivation for Vaccination Against COVID-19 among Young Adults}

One of the major challenges in the fight against COVID-19 is encouraging young people to take the vaccine to increase the chances of populations achieving herd immunity (Lazarus et al., 2020; Robertson et al., 2021). Young adults are not concerned about COVID-19 (Drążkowski \& Trepanowski, 2021; Y1ldırım et al., 2020) as this disease does not pose a great risk to their health (Kang \& Jung, 2020). Thus, for young adults, arguments aimed at vaccine uptake to protect personal health are most likely to be less convincing than arguments aimed at protecting the health of others, such as the elderly and the sick. This is supported by studies indicating that the motivation to be vaccinated is to protect the health of 
others. Recent research shows that willingness to protect others is an important motivation for vaccination behavior (Betsch et al., 2018) and that concern for others increases the intention to get vaccinated against COVID-19 (Jung \& Albarracín, 2020).

This analysis suggests that young adults' motivation to vaccinate against COVID-19 to protect others' health may be stronger than that to protect personal health. Thus, among young adults, targeting self-persuasion to protect the health of others may be more encouraging to vaccinate against COVID-19 than to protect one's own health.

Empathy. Since concern for protecting the health of others is an important motivation to vaccinate against COVID-19, it is reasonable to expect that highly empathetic individuals, who are more concerned about the welfare of others, will be more likely to vaccinate than low-empathetic individuals. Indeed, one study demonstrated that willingness to vaccinate increased as empathy increased (Pfattheicher et al., 2021). Similarly, another study demonstrated that empathy promotes the motivation to engage in physical distancing and wearing of face masks during the COVID-19 pandemic (Pfattheicher et al., 2020). Pfattheicher et al. (2021) explained that promoting empathy can increase the intention to vaccinate against COVID-19, as vaccination can be conceptualized as a prosocial act that helps to protect vulnerable others.

\section{Theory of Planned Behavior}

Identifying pathways mediating the effect of self-persuasion targeted at protecting the health of others on the intention to vaccinate will help understand the mechanism underlying this type of persuasive communications. The theory of planned behavior (TPB; Ajzen, 1991) is particularly relevant to studies on vaccination intention as it helps to identify determinants that guide intentional behavior. Thus, TPB might help to disentangle the motivation for COVID-19 vaccination intention. TPB has been widely applied to understand and predict the 
intention to vaccinate in both pre-pandemic (Agarwal, 2014) and pandemic conditions (Drążkowski \& Trepanowski, 2021; Lueck \& Spiers, 2020). Intentional behavior arises from three motivational belief systems about any given behavior: utility, social norms, and control beliefs. Utility beliefs focus on the consequences of the behavior and result in either an advantageous or disadvantageous attitude toward the behavior. Social norm beliefs are concerned with others' expectations of the behavior and establish and reinforce social norms. Control beliefs are perceptions about the difficulty or effort required to execute a behavior and deal with challenges, resulting in perceived behavioral control. All three belief systems indirectly affect behavior through intention.

Vaccination behavior has a potential, substantial impact on the welfare of others as it can protect them from contracting a severe disease and therefore can be considered a behavioral manifestation of moral norms. An individual's moral norms are defined as socially determined and validated values they attach to behaviors (Manstead, 2000). Moral beliefs can work in parallel with the TPB belief systems (Conner \& Armitage, 1998). Furthermore, moral norms significantly predict vaccination intention when controlling for all TPB components (Juraskova et al., 2012) and are highly related to vaccination intention against COVID-19 (Drążkowski \& Trepanowski, 2021).

Our research aims to identify pathways mediating the effect of self-persuasion for protecting the health of others through two studies. First, based on the TPB model (Ajzen, 1991) we expect that young adults under self-persuasion to vaccinate against COVID-19 to protect the health of others (sick, elderly) will create arguments indicating that the vaccination: 1) is effective in protecting the health of others; 2) uptake will lead to positive reactions by the others; 3 ) is morally right because it protects others; 4) easily contributes to the protection of others. We hypothesize that self-persuasion focused on others' health 
protection will influence the increase in intention to vaccinate by greater beliefs: 1) utility, 2) social norms, 3) moral norms, and 4) control (study 1 and study 2).

Second, previous studies indicate a positive association between empathy and intention to vaccinate against COVID-19 (Pfattheicher et al., 2021). As highly empathic people are primarily focused on the benefits of others rather than their own, we expect that similar mechanisms may be identified in creating arguments on vaccination focused on others' health. Thus, we hypothesize that as empathy increases, utility, social and moral norms, and control beliefs increase, which in turn are associated with a greater intention to vaccinate against COVID-19 (Study 1).

Finally, based on prior findings (Drążkowski \& Trepanowski, 2021; Schwarzinger et al., 2021), gender and knowing someone afflicted with COVID-19 could intervene into the explanation of young adults' motivation to vaccinate against COVID-19 within the TPB model. Thus, we hypothesize that, through TPB components, women are less willing to vaccinate than men, but these relationships are suppressed by the links between gender and empathy (Study 1). We also hypothesize that, as shown in a previous study (Drążkowski \& Trepanowski, 2021), knowing someone with COVID-19 will be associated with a greater intention to vaccinate through all TPB components (Study 1 and Study 2).

\section{Study 1}

Study 1 aimed to examine (1) whether self-persuasion focused on protecting others' health leads to greater intention to vaccinate compared to direct persuasion; (2) whether empathy is associated with an increased intention to vaccinate against COVID-19; (3) whether components of the TPB model (utility, control, social, and moral norm beliefs) mediate between self-persuasion and vaccination intention; and (4) the effects of gender and knowing a person with COVID-19 on vaccination intention. 


\section{Participants and Procedure}

Young Polish adults $(\mathrm{n}=366)$ participated in an online study in late September 2020, which was carried out in accordance with the Declaration of Helsinki and its amendments. The demographic data of the participants are presented in Table 1. The participants were recruited through a dissemination of short information about the study on multiple Facebook groups and communities, especially those dedicated to young people and students. All participants provided informed consent. Fourteen responses were excluded from the analyses: three exactly same questionnaires were submitted by the same participant due to a software error, and 12 participants did not complete the entire questionnaire. Therefore, 352 responses were analyzed. The data used in this study are available for free download from the Open Science Framework (OSF) ${ }^{1}$.

\section{Table 1}

Participants' demographic data (Study 1)

\begin{tabular}{lcc}
\hline Descriptive variable & $n$ & $\%$ \\
\hline Age $(M ; S D)$ & \multicolumn{1}{c}{$M=22.37 ; S D=2.23$} \\
$18-20$ & 67 & 19 \\
$21-25$ & 252 & 71.5 \\
$26-30$ & 33 & 9.5 \\
Sex & & \\
Male & 64 & 18.2 \\
Female & 280 & 79.5 \\
Other/Non-binary & 5 & 1.4 \\
No data & 3 & .9 \\
Have you gotten sick with COVID-19? & & \\
No & 203 & 57.5 \\
Yes & 3 & .9 \\
I don't know & 146 & 41.5 \\
Do you personally know someone who has gotten sick with & \\
COVID-19? & & \\
No & 165 & 46.9 \\
Yes & 136 & 38.6 \\
I don't know & 51 & 14.5 \\
\hline
\end{tabular}

\footnotetext{
${ }^{1}$ https://osf.io/ckf2v/?view only=42799b0d579b437c8cb926ccfddbfba0
} 
First, each participant was presented with short information about the study and their rights as participants. Subsequently, they were randomly assigned to one of the three possible versions of the study. At the beginning of each version of the study, participants completed a measure of empathy, after which the experimental manipulation occurred. In the direct persuasion group $(n=119)$, participants were asked to read two arguments in favor of getting vaccinated, which were created by the WHO. Participants rated the extent to which they agreed to each argument on a seven point scale $(1=I$ do not agree at all to $7=I$ completely agree). In the experimental group targeted at others' health $(n=123)$, participants were asked to create two or more arguments in favor of vaccinating to protect the health of others. In the second experimental group $(n=110)$, the participants were asked to create a similar set of arguments, although this time, in favor of vaccinating to protect their own health. Participants in both experimental groups were informed that their arguments would be rated by a group of experts to select the most persuasive ones. The participants then completed a set of measures, as described in the following section. At the end of the study, participants were asked to provide a code consisting of the first letters of their father's name, mother's name, maternal grandmother's name, and month of birth. This code would provide anonymity to all participants. All items used in the study, as well as the experimental manipulations, are available in the Supplementary Files, except for the empathy measure due to copyrights.

\section{Measures}

\section{Empathy}

We used 21 items from The Empathic Sensitiveness Scale (Kaźmierczak et al., 2007) to measure the emotional and cognitive aspects of empathy scored on a 5-point Likert scale $(1=$ completely disagree to $5=$ completely agree $)$. This scale is based on the Interpersonal Reactivity Index (Davis, 1983). 


\section{Utility Beliefs}

Judgements about the possible consequences of being vaccinated against COVID-19 were assessed using self-created three seven-point bipolar adjective scales (e.g., "unprotective/protective").

\section{Control Beliefs}

Beliefs about the degree of control over taking the vaccine against COVID-19 when it becomes available were assessed using three items (e.g., "It's mostly up to me whether I get vaccinated against COVID-19 when a vaccine becomes available") rated on self-created seven-point bipolar scales with different anchors (e.g., "How much control will you have over getting vaccinated").

\section{Social Norm Beliefs}

Beliefs about the attitudes and opinions of others, especially those important to the participant, about the participant's vaccination intention were assessed using three self-created items (e.g., "Most people who are important to me would praise me for getting vaccinated against COVID-19") rated on a seven-point scale $(1=$ completely disagree to $7=$ completely agree).

\section{Moral Norm Beliefs}

Beliefs about the participant's moral responsibilities regarding COVID-19 vaccination were assessed using three self-created items (e.g., "Getting vaccinated against COVID-19 should be a moral obligation for all people") rated on a seven-point scale ( 1 = completely disagree to $7=$ completely agree)

\section{Vaccination Intention}


Intention to vaccinate against COVID-19 when a free vaccine becomes available was assessed using three self-created items (e.g., "When the vaccine becomes available, I will vaccinate myself") rated on a seven-point scale $(1=$ completely false to $7=$ completely true $)$.

\section{Demographic Data}

The participants were asked to provide their age and sex, as well as whether they were afflicted with COVID-19 (S-COVID-19), or if they knew someone who was afflicted with COVID-19 (K-COVID-19).

\section{Analysis and Results}

We conducted structural equation modelling (SEM) in $\mathrm{R}$ with the lavaan library ( $\mathrm{R}$ Core Team, 2020; Rossel, 2012) using nonparametric statistics and estimators because of the lack of normality of the tested variables (K-S test; $\mathrm{p}<.001)$. Before the analyses, we prepared the data by: (1) searching for outliers and removing them; (2) coding sex as a binary dummy variable and removing participants, who did not report sex or reported sex other than male/female, due to insufficient number of them; (3) coding the K-COVID-19 as a binary dummy variable by combining "no" and "don't know" responses owing to low number of participants who reported that they don't know if they are acquainted with someone who was afflicted with COVID-19; (4) dummy coding the experimental group variable, in such a way, that two variables were created, allowing for comparison of those groups in the following regressions in SEM. Furthermore, the S-COVID-19 variable was not used in the analyses because of the insufficient number of "Yes" responses. Finally, 344 responses were included in further analyses.

Confirmatory factor analysis (CFA) and reliability analyses were conducted first. We used the weighted least squares mean (WLSM) estimation method, which performs well when using non-normally distributed and categorical data (Moshagen \& Musch, 2014). As 
Supplementary Table 1 shows, the control beliefs scale had one item removed since its factor loading was lower than .3. The empathy scale was also reduced by removing an item and then conducting another CFA after each removal until all items had factor loadings higher than .3. This resulted in an 11-item empathy scale that was used in further analyses. Next, we conducted reliability analyses, Spearman's rho correlation analysis, and calculated the heterotrait-monotrait ratio of correlations (HTMT; Hensler et al, 2015), which are reported in Tables 3 and 4, respectively. Table 2 also contains Cronbach's alpha reliabilities and average variances extracted (AVE), used to test for divergent validity of the measures used in the study. For this, we adopted two criteria. One, the Fornell-Larcker criterion (1981), assuming that for the divergence of the variables, AVE should not exceed the squared correlation. To simplify the calculations, we used the square root of AVE instead. Two, HTMT should not exceed .85 , or .90 , if the measured constructs are similar. For the empathy measure, we adapted the former value, while for the remaining variables, the latter. The moral norm beliefs measure was excluded from the analyses because they did not meet the HTMT criterion $\left(\mathrm{HTMT}_{\text {Moral norms-Intention }}=.94 ; \mathrm{HTMT}_{\text {Moral norms-Utility beliefs }}=.90\right)$, as reported in Table 3.

\section{Table 2}

Spearman's rho, means, standard deviations, and average variance extracted in Study 1

\begin{tabular}{|c|c|c|c|c|c|c|c|c|}
\hline & 1 & 2 & 3 & 4 & 5 & 6 & 7 & 8 \\
\hline 1. Empathy & - & & & & & & & \\
\hline 2. Intention & $.13^{*}$ & - & & & & & & \\
\hline 3. Utility beliefs & $.12 *$ & $.77^{* *}$ & - & & & & & \\
\hline 4. Control beliefs & $.17 * *$ & $.72^{* *}$ & $.68^{* *}$ & - & & & & \\
\hline 5. Social norm beliefs & .09 & $.71 * *$ & $.69^{* *}$ & $.63^{* *}$ & - & & & \\
\hline 6. Moral norm beliefs & $.13^{*}$ & $.85^{* *}$ & $.77^{* *}$ & $.69^{* *}$ & $.75^{* *}$ & - & & \\
\hline 7. $\mathrm{Sex}$ & $-.25^{* *}$ & .03 & .08 & -.01 & .06 & .03 & - & \\
\hline 8. K-COVID- 19 & .06 & $.15^{* *}$ & $.16^{* *}$ & $.12 *$ & $.18^{* *}$ & $.12 *$ & .09 & - \\
\hline$M$ & 3.40 & 5.20 & 5.38 & 5.17 & 4.76 & 5.01 & - & - \\
\hline$S D$ & .68 & 1.95 & 1.57 & 1.50 & 1.83 & 1.89 & - & - \\
\hline$\alpha$ & .81 & .96 & .92 & .77 & .96 & .94 & - & - \\
\hline$A V E$ & .31 & .90 & .80 & .61 & .88 & .84 & - & - \\
\hline$\sqrt{ } A V E$ & .56 & .95 & .89 & .78 & .94 & .91 & - & - \\
\hline
\end{tabular}


Table 3

Heterotrait-monotrait ratio of correlations for Study 1

\begin{tabular}{lllllll}
\hline & 1 & 2 & 3 & 4 & 5 & 6 \\
\hline 1. Empathy & - & & & & & \\
2. Intention & .17 & - & & & & \\
3. Control beliefs & .25 & .86 & - & & \\
4. Utility beliefs & .15 & .88 & .86 & - & \\
5. Social norm beliefs & .14 & .81 & .77 & .79 & - & \\
6. Moral norm beliefs & .21 & .94 & .85 & .90 & .84 & - \\
\hline
\end{tabular}

Next, we performed SEM (WLSM). The initial model evaluating vaccination intention consisted of four observed exogenous variables (experimental group: self, experimental group: others, sex, K-COVID-19) and five endogenous latents (empathy, vaccination intention, control beliefs, utility beliefs, social norm beliefs, moral norm beliefs). K-COVID-19 was removed from the final model due to a lack of significance. We also estimated the error covariance among utility, social norms, and control beliefs, as well as between the experimental group variables. The factor loadings for every item included in the final model are presented in Supplementary Table 1, while the simplified model with only significant values is presented in Figure 1.

\section{Table 4}

Fit measures for Study 1

\begin{tabular}{lcc}
\hline Measure & Estimate & Cutof* \\
\hline Tucker Lewis Index (TLI) & .969 & $>.90$ \\
Comparative Fit Index (CFI) & .973 & $>.90$ \\
Goodness of Fit Index (GFI) & .995 & $>.90$ \\
Adjusted Goodness of Fit Index (AGFI) & .994 & $>.90$ \\
Root Mean Square Error of Approximation (RMSEA) & .051 & $<.08$ \\
Standardized Root Mean Square Residual (SRMR) & .060 & $<.08$ \\
\hline
\end{tabular}

*Hu, \& Bentler, 1999; Steiger, 2007 
The developed model has good fit indices, indicating that it fits the data well. We used the robust versions of the following indices: Tucker-Lewis index (TLI), comparative fit index (CFI), goodness of fit index (GFI), adjusted goodness of fit index (AGFI), root mean square of error of approximation (RMSEA), and standardized root mean square residual (SRMR), values of which are reported in Table 4 alongside the suggested cutoff lines. $\mathrm{Chi}^{2}$ was significant even with the Satorra-Bentler correction $\left(c h i^{2}=745.06, d f=278, \mathrm{p}<.001\right)$.

\section{Figure 1}

Simplified SEM model for Study 1 with only significant values

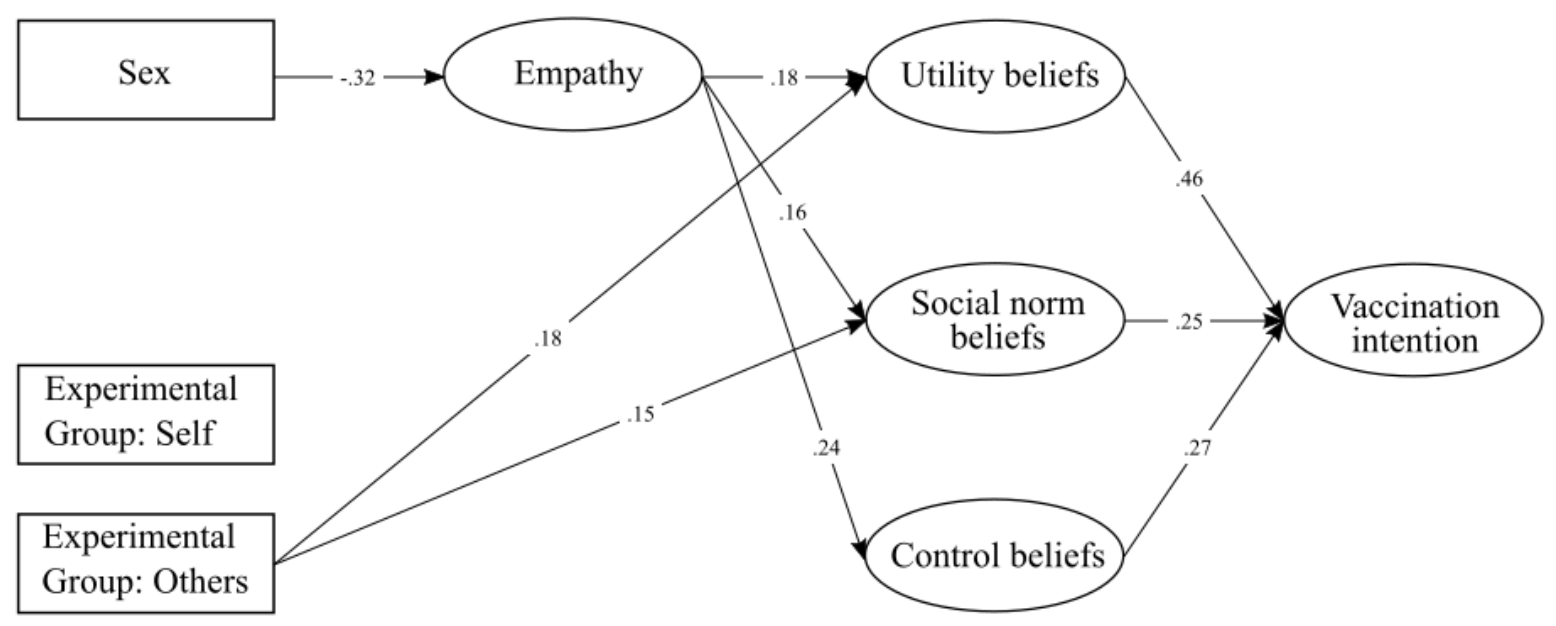

As shown in the model, the experimental manipulation focused on protecting the health of others had a significant effect on utility beliefs $(\beta=.18)$ and social norm beliefs $(\beta$ $=.15)$, while empathy had a significant effect on all TPB components $\left(\beta_{\text {utility beliefs }}=.18, \beta_{\text {social }}\right.$ beliefs $\left.=.16, \beta_{\text {control beliefs }}=.24\right)$. Simultaneously, being affected by sex, females were more likely to have reached high empathy values $(\beta=-.32)$. Furthermore, all TPB components had a significant effect on vaccination intention $\left(\beta_{\text {utility beliefs }}=.46, \beta_{\text {social beliefs }}=.25, \beta_{\text {control beliefs }}=.27\right)$. There were also some noteworthy indirect effects, such as the effect of the experimental group: others on vaccination intention through utility beliefs $(\beta=.81)$ or the effect of sex on 
control beliefs through empathy $(\beta=-.79)$. The remaining indirect, direct, and total effects are reported in Supplementary Table 2.

\section{Discussion}

As expected, the results of Study 1 demonstrated that among young adults, self-persuasion focused on others' health (compared to self-persuasion targeted at one's own health and to direct persuasion) had an indirect effect on vaccination intention against COVID-19 through utility and social norms beliefs. As hypothesized, empathy increases, utility, social norms, and control beliefs increase, which in turn is associated with a greater intention to vaccinate against COVID-19. Moreover, gender did not significantly influence either the intention to vaccinate or TPB components. Women were more empathetic than men, and through their relationship with empathy, being female was associated with more positive attitudes towards vaccination and greater perceived behavioral control. Finally, knowing someone with COVID-19 was unrelated to the intention to vaccinate and all TPB components.

\section{Study 2}

A limitation of Study 1 was the difficulty of using self-persuasion to encourage young people to vaccinate against COVID-19 on a large scale. A method of inducing self-persuasion that overcomes these limitations is using open-ended questions that can effectively encourage specific health behaviors (Glock et al., 2013; Müller et al., 2016). Prior studies on the effectiveness of posters on alcohol consumption demonstrated that messages framed as open-ended questions trigger self-generation of arguments for drinking less alcohol, which subsequently reduces actual alcohol consumption among young adults (Loman et al., 2018). 
This study also showed that messages framed as open-ended questions evoked a less reactive state than direct persuasion, which may explain the effectiveness of self-persuasion in changing unhealthy behavior. The great advantage of messages framed as open-ended questions is that they are both effective and simple.

Similar to Study 1, we used self-persuasion targeted at protecting the health of others. In line with study 1, we aimed to test whether components of the TPB model (utility, control, social, and moral norm beliefs) mediate between self-persuasion and intention to vaccinate and examine the effects of gender and knowing a person with COVID-19 on intention to vaccinate. Thus, the aim of Study 2 is to apply the results from study 1, using self-persuasion activated by the poster with messages framed as open-ended questions, compared with direct persuasion, could effectively encourage vaccination against COVID-19 among young adults.

\section{Participants and procedure}

A total of 375 Polish adults participated in the second study in October 2020. Of them, most were women $(70.7 \%)$. No responses were removed. Again, the participants were recruited through dissemination of study invitations on Facebook. All participants provided informed consent. The data used in the study are available at the OSF, while the specific demographics of the participants are presented in Table 5.

\section{Table 5}

Participants'demographic data (Study 2)

\begin{tabular}{lcc}
\hline Descriptive variable & $n$ & $\%$ \\
\hline Age $(M ; S D)$ & & $M=21.07 ; S D=2.19$ \\
$18-20$ & 182 & 48.5 \\
$21-25$ & 178 & 47.5 \\
$26-30$ & 15 & 4 \\
Sex & & \\
Male & 106 & 28.3 \\
Female & 265 & 70.7 \\
Other/Non-binary & 4 & 1.1 \\
No data & 0 & 0
\end{tabular}


Have you gotten sick with COVID-19?

$\begin{array}{lcc}\text { No } & 215 & 57.3 \\ \text { Yes } & 3 & .8 \\ \text { I don't know } & 157 & 41.9 \\ \begin{array}{l}\text { Do you personally know someone who has } \\ \text { gotten sick with COVID-19? }\end{array} & \\ \text { No } & 162 & 43.3 \\ \text { Yes } & 171 & 45.6 \\ \text { I don't know } & 42 & 11.2\end{array}$

Participants were randomly assigned to one of the two groups. In both groups, participants were asked to choose an image from a set of two that they thought best persuaded them to take the vaccine. In addition, the participants were told that the questionnaire consisted of two different studies. The aim of the "first" study was to create a graphic that effectively persuades people to take the vaccine, while the aim of the "second" study was to explore factors leading to vaccination.

In the direct persuasion group two images were exposed included young people and an imperative sentence, encouraging people to protect their own health through vaccinating ("Protect yourself and your own health - take the coronavirus vaccine!"). In the focusing on protecting others' health group, participants were exposed with two similar images of older people, both supplied with a short question written on them, which was supposed to encourage them to take the vaccine to protect the health of others ("How can you protect other people, especially those who cannot take the vaccine due to health problems, through vaccinating?"). Participants subsequently completed a short questionnaire including the same measures as in Study 1, except for the empathy questionnaire, which was not used in this study. The images used in the study were taken from the Twitter of the Polish Ministry of Health $^{2}$ and WHO website ${ }^{3}$, and then edited so that only old or young people were present in

\footnotetext{
${ }^{2}$ https://twitter.com/MZ GOV PL/status/1243787403958947840 (Accessed September/October 2020) 3https://www.who.int/bangladesh/emergencies/coronavirus-disease-(covid-19)-update/advice-for-public---communicating-se verity-series/images/default-source/searo---images/countries/bangladesh/infographics/risk-comms/english/slide5?itemIndex $=5$ (Accessed September/October 2020)
} 
the image. Original images are available at footnote, while the edited images used in the study are attached in the Supplementary Files.

\section{Analysis and Results}

We conducted SEM using the same procedure as in Study 1. The CFA (Supplementary Table 3) indicated that two items belonging to the control scale had low factor loadings. Consequently, they were removed. The divergent validity analyses (Table 6; Table 7) indicated that the moral norm and utility belief measures did not meet the HTMT criterion $\left(\mathrm{HTMT}_{\text {Moral norms-Intention }}=.93 ; \mathrm{HTMT}_{\text {Utility beliefs-Intention }}=.91\right)$.

\section{Table 6}

Spearman's rho, means, standard deviations, and average variance extracted in Study 2

\begin{tabular}{|c|c|c|c|c|c|c|c|}
\hline & 1 & 2 & 3 & 4 & 5 & 6 & 7 \\
\hline 1. Intention & $\overline{-}$ & & & & & & \\
\hline 2. Utility beliefs & $.80 * *$ & - & & & & & \\
\hline 3. Control beliefs & $.35^{* *}$ & $.33^{* *}$ & - & & & & \\
\hline 4. Social norm beliefs & $.65^{* *}$ & $.65^{* *}$ & $.24^{* *}$ & - & & & \\
\hline 5. Moral norm beliefs & $.84^{* *}$ & $.80^{* *}$ & $.32 * *$ & $.73^{* *}$ & - & & \\
\hline 6. $S e x$ & .03 & $.11^{*}$ & -.00 & .08 & .06 & - & \\
\hline 7. K-COVID-19 & .09 & .10 & -.00 & .10 & $.12 *$ & -.02 & - \\
\hline$M$ & 5.08 & 5.30 & 5.59 & 4.47 & 4.97 & - & - \\
\hline$S D$ & 1.92 & 1.63 & 1.04 & 1.77 & 1.90 & - & - \\
\hline$\alpha$ & .95 & .92 & .54 & .95 & .95 & - & - \\
\hline$A V E$ & .88 & .82 & .38 & .87 & .87 & - & - \\
\hline$\sqrt{ } A V E$ & .93 & .90 & .62 & .93 & .93 & - & - \\
\hline \multicolumn{8}{|c|}{$\begin{array}{l}\text { Note. }{ }^{*} p<.05,{ }^{* *} p<.01,{ }^{* * *} p<.001 \text {, Sex was dummy coded as } 0=\text { Female, } 1=\text { Male, while K-COVID-19 as } 0=\text { No, } 1= \\
\text { Yes }\end{array}$} \\
\hline \multicolumn{8}{|l|}{ Table 7} \\
\hline \multicolumn{8}{|c|}{ Heterotrait-monotrait ratio of correlations for Study 2} \\
\hline & 1 & & & 3 & & & \\
\hline 1. Intention & - & & & & & & \\
\hline 2. Control beliefs & .78 & & & & & & \\
\hline 3. Utility beliefs & .91 & & & - & & & \\
\hline 4. Social norm beliefs & .73 & & & .72 & & & \\
\hline 5. Moral norm beliefs & .93 & & & .89 & & & \\
\hline
\end{tabular}


The initial model for this study comprised three exogenous observed variables (sex, K-COVID-19, group) and three endogenous latents (control beliefs, social norm beliefs, vaccination intention). The K-COVID-19 variable was removed from the final model because of its lack of significance. The factor loadings are presented in Supplementary Table 3, and a simplified model with only significant values is presented in Figure 2.

\section{Figure 2}

Simplified SEM model for Study 2 with only significant values

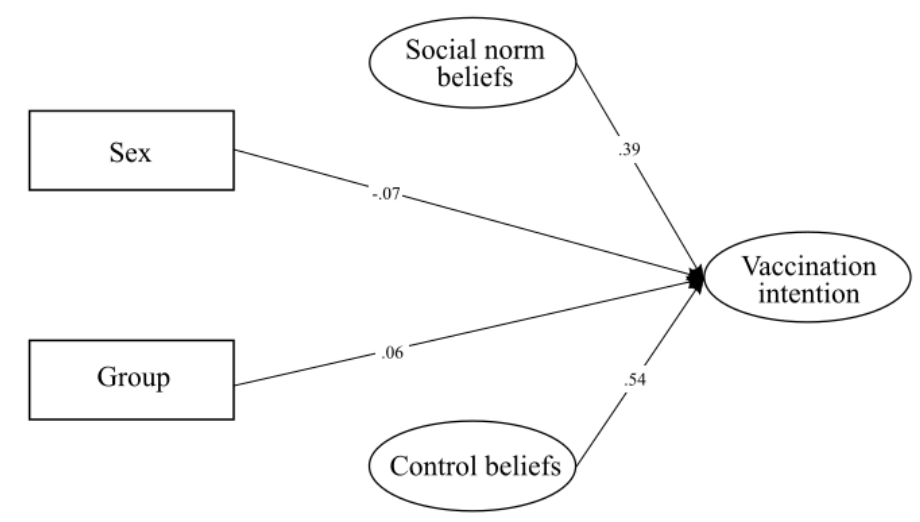

As reported in Table 8, the developed model had good fit indices, although $\mathrm{Chi}^{2}$ with the Satorra-Bentler correction was significant $\left(c h i^{2}=60.081, d f=27, \mathrm{p}<.001\right)$. Nevertheless, the model fits the data well. Results indicated that all variables had significant direct effects on vaccination intention $\left(\beta_{\text {control beliefs }}=.54 ; \beta_{\text {social norm beliefs }}=.39 ; \beta_{\text {sex }}=-.07 ; \beta_{\text {group }}=.27\right)$. These results indicate that women were more likely to have higher vaccination intention, similar to people who were assigned to the experimental group focused on protecting the health of others. In accordance with the data presented in Supplementary Table 2, no significant indirect or total effects were detected.

\section{Table 8}

Fit measures for Study 2 


\begin{tabular}{llc}
\hline Tucker Lewis Index (TLI) & .998 & $>.90$ \\
Comparative Fit Index (CFI) & .999 & $>.90$ \\
Goodness of Fit Index (GFI) & 1.000 & $>.90$ \\
Adjusted Goodness of Fit Index (AGFI) & 1.000 & $>.90$ \\
Root Mean Square Error of Approximation (RMSEA) & .019 & $<.08$ \\
Standardized Root Mean Square Residual (SRMR) & .018 & $<.08$ \\
\hline
\end{tabular}

*Hu, \& Bentler, 1999; Steiger, 2007

\section{Discussion}

The results of Study 2 indicated the potential for large-scale application of self-persuasion to encourage young adults to vaccinate against COVID-19. We found that a poster with messages framed as open-ended questions provoking self-persuasion targeted at others' health could influence the intention to vaccinate against COVID-19. Because of the strong correlation between utility beliefs and intentions, we had to remove this variable from the model.

Thus, the model in Study 1 was fundamentally different from that in Study 2. In contrast to Study 1, wherein the effect of self-persuasion was mediated by attitude and norms, in Study 2, the effect of self-persuasion on increased intention to vaccinate was direct. In Study 2, gender affected intention directly-women were more likely to be vaccinated against COVID-19 than men. As in Study 1, the link between moral norms and intention in Study 2 was too strong to include moral norms in the model, while knowing someone with COVID-19 was not a significant predictor of intention to vaccinate.

\section{General Discussion}

First of all, young adults could be encouraged to vaccinate against COVID-19 by self-persuasion focused on others' health. Self-persuasion through both writing out arguments (Study 1) or being exposed to a poster with open-ended questions (Study 2) led to greater 
vaccination intention than direct persuasion. Thus, we demonstrated that self-persuasion consistently outperformed its direct persuasion counterpart. We also found that highly empathetic young adults have a higher intention to vaccinate because they have higher utility, control, and social beliefs (Study 1).

Our findings demonstrated that among young adults, self-persuasion targeted at others' health leads to higher vaccination intention against COVID-19 than self-persuasion targeted at one's own health (Study 1) and direct persuasion (Studies 1 and 2). This finding is consistent with studies showing that self-persuasion can increase the intention to vaccinate against HPV (Baldwin et al., 2017). Based on previous research findings, we explain the effectiveness of self-persuasion, compared to direct persuasion, as follows: self-persuasion leads to less reactance than direct persuasion (Mussweiler \& Neumann, 2000; Wilson \& Brekke, 1994); (2) self-generated arguments arouse the need to reduce cognitive dissonance by changing one's attitude (Festinger, 1957); and (3) self-generated arguments were tailored to people's concerns to a greater extent than arguments from direct persuasion (Baldwin et al., 2013).

1.1. Study 1. Self-persuasion targeted at protecting one's own health was less effective in encouraging vaccination than self-persuasion targeted at protecting the health of others among young adults. Thus, it was crucial for young adults to target the arguments created in self-persuasion so that they were related to protecting the health of others - the elderly and the sick. We argue that since COVID-19 does not pose a great risk to young adults' health (Kang \& Jung, 2020), they do not perceive COVID-19 as a severe disease (Drążkowski \& Trepanowski, 2021; Y1ldırım et al., 2020), therefore self-generated arguments aimed at protecting personal health are less convincing for young adults to vaccinate than arguments aimed at protecting the health of others. Previous studies have already shown that concern for 
the health protection of others is an important motivation for vaccination (Betsch et al., 2018; Jung \& Albarracín, 2020). Thus, our findings contribute to previous works that have conceptualized vaccination as a prosocial behavior (e.g., Betsch et al., 2018; Chapman et al., 2012; Pfattheicher et al., 2021).

Study 1 expands those works by showing that: 1) self-persuasion targeting prosocial aspects of vaccination can increase motivation to vaccinate; 2) motivation based on prosocial aspects of vaccination is stronger among young people than motivation based on personal health benefits. Our findings showed that among young adults, self-persuasion targeted at protecting others' health was more effective in encouraging vaccination than self-persuasion targeted at protecting one's own health. This finding contradicts the results of Ashworth et al. (2021). Their study demonstrated that direct persuasion focused on personal health benefits is a more effective approach to enhance COVID-19 vaccination intention than direct persuasion, focusing on others' health benefits. However, this study was conducted on the general population, and therefore the results cannot be generalized to young adults, who are not concerned about personal health (Laaksonen et al., 2003).

1.2. TBP. By applying TPB, our results provide unique insights into the mechanism by which self-persuasion influences change in intention to vaccinate. First, consistent with previous findings (Drążkowski \& Trepanowski, 2021; Lueck \& Spiers, 2020), we demonstrated that utility, control, and social norm beliefs significantly predict the intention to vaccinate against COVID-19. Moral norms were found to be strongly associated with the intention to vaccinate to be included as a separate variable in the examined models. Such a strong relationship between the variables supports the idea that people perceive vaccinating against COVID-19 as a strongly moral, prosocial behavior. Second, we found that self-persuasion targeted at others' health (compared to self-persuasion targeted at one's own 
health and to direct persuasion) has an indirect effect on vaccination intention against COVID-19 through utility and social norms beliefs. We argue that young adults under self-persuasion targeted to protect others' health created arguments indicating that vaccination is effective in protecting the health of others, which increases their utility beliefs. An example of the arguments given by participants is that: "The fact that someone has a strong immune system is a very selfish way of looking at a pandemic, it's not about you it's about others so you should be vaccinated, not just so that you do not get sick but so that other people around you do not get hurt". Further, we consider that creating arguments indicating vaccination benefits for others reinforced the belief that other people expect them to vaccinate, which influenced the increase in their social norm beliefs. The lack of effect of self-persuasion on control beliefs suggests that arguments indicating that vaccination is an easy way to contribute to the protection of others did not arise in response to targeted self-persuasion. Overall, the present findings contribute to the existing literature by identifying pathways from TPB mediating the effect of self-persuasion targeted at protecting the health of others on vaccination intention. This supports our understanding of the mechanism underlying the intervention.

1.3. Empathy. The result demonstrating that self-persuasion focused on protecting the health of others can be effective in increasing young people's willingness to vaccinate, which is closely correlated with our finding that as empathy increases, the intention to vaccinate increases. This finding is consistent with previous studies indicating that vaccinating oneself is a prosocial behavior; therefore, empathic concern motivates people to protect the health of others by vaccinating (Pfattheicher et al., 2021). Highly empathic people have an other-directed focus of attention, which manifests in seeking information about the needs of others (Andreychik \& Lewis, 2017). Thus, it is typical for highly empathetic people to focus 
on protecting the health of others when making decisions to vaccinate themselves. These explanations are consistent with the results of studies showing that concern for others increases the intention to get vaccinated against COVID-19 (Jung \& Albarracín, 2020). Our results support previous findings showing that heightening empathy leads to an increase in the intention to vaccinate against COVID-19. It is possible that self-persuasion focused on protecting the health of others also leads to an increase in empathy, which may be one of the key mechanisms explaining the relationships observed in our study. Future research can empirically test this possibility by incorporating the measurement of state empathy following the use of self-persuasion aimed at protecting the health of others.

Our findings provide a deeper understanding of the relationship between empathy and intention to vaccinate through the application of TPB. Thus, we extend previous studies (Pfattheicher et al., 2021) by showing that empathy is linked with greater intention to vaccinate through a more positive attitude toward vaccination, stronger social norm beliefs related to vaccination and increased perceived behavioral control. The positive association between empathy and TPB components may be due to the tendency of highly empathic people to focus on the well-being of others (Andreychik \& Lewis, 2017). This leads them to perceive great benefits of vaccination for others, to judge that close people expect them to be vaccinated against COVID-19, and to judge that vaccination is an easy and low-effort way to help others.

1.4. Gender. Contrary to previous studies (Drążkowski \& Trepanowski, 2021; Ruiz \& Bell, 2021) and our hypothesis, men had no greater intention to be vaccinated than women. In Study 1, we found no significant gender differences in vaccination intention, and in Study 2, women were more likely to be vaccinated against COVID-19 than men. In Study 1, women were more empathetic than men, and through their relationship with empathy, being female 
was associated with more positive attitudes towards vaccination and greater perceived behavioral control. The relationships between gender and the intention to vaccinate against COVID-19 appear to be complex and sample-specific. Possibly, we failed to replicate previous findings because gender differences in motivation to vaccinate were smaller among the young adult group.

1.5. Study 2. The results of Study 2 indicated that self-persuasion could be used on a large scale. A poster with messages framed as open-ended questions effectively encouraged vaccination against COVID-19 among young adults compared to a poster containing direct persuasion. As in Study 1, we targeted self-persuasion to protect the health of others by presenting participants with the following question: "How can you protect other people, especially those who cannot take the vaccine due to health problems, through vaccinating?". Based on previous studies (Loman et al., 2018), we believe that open-ended questions trigger self-generation of arguments for why vaccination against COVID-19 can protect others' health, subsequently enhancing vaccination intention. Further, we argue that our message framed as an open-ended question evoked a lower reactance state than direct persuasion, which may partly explain the effectiveness of self-persuasion targeted at protecting others' health in promoting vaccination intention.

The current findings may have important implications for research on self-persuasion. The present work extends the classic work on self-persuasion by Aronson (1999) and shows that self-persuasion can be targeted, that is, it can target what kind of arguments will be generated. In Study 1, we showed that targeting self-persuasion to protect the health of others is more effective in encouraging vaccination than targeting self-persuasion to protect one's own health. The differences in the effectiveness of the two forms of targeted self-persuasion demonstrate that targeting self-persuasion can be controlled. Thus, our results show the 
possibility of increasing the effectiveness of self-persuasion by addressing the content of generated arguments. Our findings open new avenues of research that can test what kinds of self-generated arguments can more effectively persuade people to engage in specific health behaviors. Targeted self-persuasion can be adopted for interventions concerned with other health behaviors, such as reducing smoking and alcohol consumption, or promoting healthy eating.

1.6. Inputs of open-ended questions for mass media communication. Since our findings show that self-persuasion is more effective than direct persuasion, large-scale application of our findings might be beneficial. Study 2 findings suggest that self-persuasion can be successfully applied to media communications by framing health messages as open-ended questions. The great advantage of messages framed as open-ended questions is that they are both effective and simple; therefore, they could be applied in persuasive media messages encouraging COVID-19 vaccination. Self-persuasion by open-ended questions can be placed in various contexts: in health campaigns on television, radio, in the press, on the Internet, in interactions between health education practitioners and young people, or between doctors and patients. Self-persuasion seems to be effective only when people experience full freedom to choose their behavior (Loman et al., 2019). Thus, practitioners should avoid pushing people too hard (through rewards or punishment) to create an argument for COVID-19 vaccination. Caution should also be exercised as self-persuasion receivers should have knowledge about vaccination to be able to generate arguments as to why vaccination can protect others' health. Our study sample mostly included students and were likely to have the knowledge necessary to create arguments. For less educated groups, it seems that self-persuasion could be combined with education about vaccination. 
1.7. Proximity with COVID-19. Contrary to previous studies (Drążkowski \& Trepanowski, 2021; Schwarzinger et al., 2021) and our hypothesis, we found that among young adults, knowing someone afflicted with COVID-19 was not significantly correlated with intention to vaccinate against COVID-19 and with TPB components. It may be that during the study period, young adults did not have as much direct contact with severe cases of COVID-19 as older people. It may be argued that more acquaintances of older people than young people suffered from severe infection with COVID-19. Thus, limited contact with severely ill people with COVID-19 may have influenced the lack of a significant relationship between knowing someone afflicted with COVID-19 and the intention to vaccinate.

\section{Limitations}

Several limitations of this study need to be considered. First, the participants of our study were Facebook users, which may limit the generalizability of the results. Our sample was recruited through social media, which could have affected its bias. For example, the population we sampled mainly completed higher education. Further studies might test if the same models hold for young adults with lower education. Second, our study did not measure actual vaccination behavior because the timing of the studies preceded the widespread availability of COVID-19 vaccines. We measure the intention to vaccinate in the future. Notably, prior research (Randall \& Wolff, 1994) strongly indicates that the intention of a given behavior is a significant predictor of actual future behavior. Therefore, prospective studies should also incorporate measures of actual behavior. Third, in Study 2, the control beliefs scale consisted of only two items, while it is a good practice to have at least three items per scale. This also affected the reliability of the scale. In future studies, this scale will be revised. Finally, caution is required when applying the results of this study to other cultures. The results of previous research suggest that self-persuasion may be a more 
effective form of convincing than direct persuasion in countries with an individualistic culture than in countries with a collectivistic culture (Li et al., 2020). In contrast, targeting persuasion to protect the health of others through vaccination may be less effective in countries with individualistic cultures than in collectivistic cultures. Support for this assumption comes from research showing a positive relationship between collectivism and others worries and concerns about COVID-19, with no significance of such a relationship in the case of individualism (Germani et al., 2020)

\section{Conclusion}

We advanced the understanding of the interplay between self-persuasion and motivational factors that gave rise to the motivation to vaccinate against COVID-19 among young adults. Our results have enhanced the existing knowledge of the relationship between self-persuasion and vaccination intention. It indicated that among young adults, self-persuasion targeted at protecting others' health, compared to direct persuasion, can affect declared vaccination intention through utility and social norms beliefs. Our results provide insight into "why" people are motivated to vaccinate against COVID-19, and accordingly contribute to the scientific pursuit of encouraging young adults to get vaccinations. Finally, our research has not only theoretical but also practical consequences, as knowing that self-persuasion can affect declared intention towards vaccination can help to effectively encourage young adults to vaccinate against COVID-19.

Encouraging young people to vaccinate is difficult as they do not perceive COVID-19 as a severe disease to the extent that older people do (Drążkowski \& Trepanowski, 2021; Y1ldırım et al., 2020). Thus, evidence-based guidelines are needed to attract and motivate young adults in the most efficient and effective ways. Taken together, the findings contribute 
to the list of evidenced-based methods of encouraging vaccination against COVID-19. Therefore, they could have important implications for ongoing government interventions via mass media designed to encourage vaccination among young adults.

\section{References}

Ajzen, I. (1991). The theory of planned behavior. Organizational Behavior and Human Decision Processes, 50, $179-211$. doi: $10.1016 / 0749-5978(91) 90020-\mathrm{T}$

Andreychik, M. R., \& Lewis, E. (2017). Will you help me to suffer less? How about to feel more joy? Positive and negative empathy are associated with different other-directed motivations. Personality and Individual Differences, 105, 139-149. doi:10.1016/j.paid.2016.09.038

Agarwal, V. (2014). A/H1N1 vaccine intentions in college students: An application of the theory of planned behavior. Journal of American College Health, 62(6), 416-424. doi:10.1080/07448481.2014.917650.

Aronson, E. (1999). The power of self-persuasion. The American Psychologist, 54(11), 875-884. https://doi.org/10.1037/h0088188

Ashworth, M., Thunström, L., Cherry, T. L., Newbold, S. C., \& Finnoff, D. C. (2021). Emphasize personal health benefits to boost COVID-19 vaccination rates. Proceedings of the National Academy of Sciences, 118(32). doi: $10.1073 /$ pnas. 2108225118

Baldwin, A. S., Denman, D. C., Sala, M., Marks, E. G., Shay, L. A., Fuller, S., ... Tiro, J. A. (2017). Translating self-persuasion into an adolescent HPV vaccine promotion intervention for parents attending safety-net clinics. Patient Education and Counseling, 100(4), 736-741. doi:10.1016/j.pec.2016.11.014

Baldwin, A. S., Rothman, A. J., Vander Weg, M. W., \& Christensen, A. J. (2013). Examining causal components and a mediating process underlying self-generated health arguments for exercise and smoking cessation. Health Psychology, 32(12), 1209. doi: 10.1037/a0029937

Betsch, C., Schmid, P., Heinemeier, D., Korn, L., Holtmann, C., \& Böhm, R. (2018). Beyond confidence: Development of a measure assessing the 5C psychological antecedents of vaccination. PLOS ONE, 13(12), e0208601. doi:10.1371/journal.pone.0208601

Conner, M., \& Armitage, C. J. (1998). Extending the theory of planned behavior: A review and avenues for further research. Journal of Applied Social Psychology, 28(15), 1429-1464.doi:10.1111/j.1559-1816.1998.tb01685.x

Chapman, G. B., Li, M., Vietri, J., Ibuka, Y., Thomas, D., Yoon, H., \& Galvani, A. P. (2012). Using game theory to examine incentives in influenza vaccination behavior. Psychological Science, 23(9), 1008-1015. https://doi.org/10.1177/0956797612437606

Drążkowski, D., \& Trepanowski, R. (2021). Reactance and Perceived Severity of a Disease as the Determinants of COVID-19 Vaccination Intention: an Application of the Theory of Planned Behavior. Psychology, Health \& Medicine (in press). https://doi.org/10.1080/13548506.2021.2014060. 


\section{VACCINATION: SELF-PERSUASION \& EMPATHY30}

Drążkowski, D., Trepanowski, R., Chwiłkowska, P., \& Majewska, M. (2020). Self-persuasion increases motivation for social isolation during the COVID-19 pandemic through moral obligation. Social Psychological Bulletin, 15(4), 1-20. doi: $/ 10.32872 / \mathrm{spb} .4415$.

Festinger, L. (1957). A theory of cognitive dissonance (Vol. 2). Stanford, CA, USA: Stanford University Press.

Fornell, C., \& Larcker, D. F. (1981). Structural equation models with unobservable variables and measurement error: Algebra and statistics. Journal of Marketing Research, 18(3), 382-388. Doi: 10.1177/002224378101800313.

Germani, A., Buratta, L., Delvecchio, E., \& Mazzeschi, C. (2020). Emerging adults and COVID-19: the role of individualism-collectivism on perceived risks and psychological maladjustment. International journal of environmental research and public health, 17(10), 3497. doi: 10.3390/ijerph17103497.

Glock, S., Müller, B. C. N., \& Ritter, S. M. (2013). Warning labels formulated as questions positively influence smoking-related risk perception. Journal of Health Psychology, 18, 252-262. doi: 10.1177/1359105312439734

Grandpre, J., Alvaro, E. M., Burgoon, M., Miller, C. H., \& Hall, J. R. (2003). Adolescent reactance and anti-smoking campaigns: A theoretical approach. Health Communication, 15(3), 349-366. https://doi.org/10.1207/S15327027HC1503_6

Henseler, J., Ringle, C. M., Sarstedt, M. (2015). A new criterion for assessing discriminant validity in variance-based structural equation modelling. Journal of the Academy of Marketing Science, 43(1), 115-135. doi: 10.1007/s11747-014-0403-8.

Hu, L., \& Bentler, P. M. (1999). Cutoff criteria for fit indexes in covariance structure analysis: Conventional criteria versus new alternatives. Structural Equation Modeling: A Multidisciplinary Journal, 6(1), 1-55. doi: $10.1080 / 10705519909540118$

Jung, H., \& Albarracín, D. (2021). Concerns for others increases the likelihood of vaccination against influenza and COVID-19 more in sparsely rather than densely populated areas. Proceedings of the National Academy of Sciences, 118(1). doi: 10.1073/pnas.2007538118

Juraskova, I., O’Brien, M., Mullan, B., Bari, R., Laidsaar-Powell, R., \& McCaffery, K. (2012). HPV Vaccination and the Effect of Information Framing on Intentions and Behaviour: An Application of the Theory of Planned Behaviour and Moral Norm. International Journal of Behavioral Medicine, 19(4), 518-525. doi:10.1007/s12529-011-9182-5

Kachurka, R., Krawczyk, M., \& Rachubik, J. (2021). Persuasive Messages Will Not Increase COVID-19 Vaccine Acceptance: Evidence from a Nationwide Online Experiment. Vaccines, 9(10), 1113. doi: 10.3390/vaccines9101113.

Kang, S. J., \& Jung, S. I. (2020). Age-related morbidity and mortality among patients with COVID-19. Infection \& chemotherapy, 52(2), 154. doi: 10.3947/ic.2020.52.2.154.

Kaźmierczak, M., Plopa, M., \& Retowski, S. (2007). Skala wrażliwości empatycznej. Przegląd Psychologiczny, 50(1), 9-24.

Laaksonen, M., Prättälä, R., \& Lahelma, E. (2003). Sociodemographic determinants of multiple unhealthy behaviours. Scandinavian Journal of Public Health, 31(1), 37-43. doi:10.1080/14034940210133915

Lazarus, J. V., Wyka, K., Rauh, L., Rabin, K., Ratzan, S., Gostin, L. O., .. \& El-Mohandes, A. (2020). Hesitant or not? The association of age, gender, and education with potential acceptance of a COVID-19 vaccine: A country-level analysis. Journal of Health Communication, 25(10), 799-807. doi: $10.1080 / 10810730.2020 .1868630$

Li, S., van Baaren, R. B., \& Müller, B. C. N. (2020). The influence of culture and close others on the effectiveness of (self)-persuasion. The Journal of General Psychology, 1-30. doi:10.1080/00221309.2020.1803193 


\section{VACCINATION: SELF-PERSUASION \& EMPATHY31}

Loman, J. G., de Vries, S. A., Kukken, N., van Baaren, R. B., Buijzen, M., \& Müller, B. C. (2019). Quick question or intensive inquiry: The role of message elaboration in the effectiveness of self-persuasive anti-alcohol posters. Plos one, 14(1), e0211030. doi: 10.1371/journal.pone.0211030

Loman, J. G. B., Müller, B. C. N., Oude Groote Beverborg, A., van Baaren, R. B., \& Buijzen, M. (2018). Self-persuasion in media messages: Reducing alcohol consumption among students with open-ended questions. Journal of Experimental Psychology: Applied, 24(1), 81-91. doi: 10.1037/xap0000162

Lueck, J. A., \& Spiers, A. (2020). Which Beliefs Predict Intention to Get Vaccinated against COVID-19? A Mixed-Methods Reasoned Action Approach Applied to Health Communication. Journal of Health Communication, 25(10), 790-798. doi: 10.1080/10810730.2020.1865488

Manstead, A. S. R. (2000). The role of moral norm in the attitude-behavior relation. In D. J. Terry \& M. A. Hogg (Eds.), Attitudes, behavior, and social context: The role of norms and group membership. Applied social research (pp. 11-30). Mahwah, NJ: Lawrence Erlbaum Associates, Publishers.

Moshagen, M., \& Musch, J. (2014). Sample size requirements of the robust weighted least squares estimator for confirmatory factor analysis with ordinal data. Methodology: European Journal of Research Methods for the Behavioral and Social Sciences, 10, 60-70. Doi: 10.1027/1614-2241/a00006.

Müller, B. C. N., Ritter, S. M., Glock, S., Dijksterhuis, A., Engels, R. C. M. E., \& van Baaren, R. B. (2016). Smoking-related warning messages formulated as questions positively influence short-term smoking behaviour. Journal of Health Psychology, 21, 60 - 68. doi: 10.1177/1359105314522083

Mussweiler, T., \& Neumann, R. (2000). Sources of Mental Contamination: Comparing the Effects of Self-Generated versus Externally Provided Primes. Journal of Experimental Social Psychology, 36(2), 194-206. doi:10.1006/jesp.1999.1415

Nyhan, B., Reifler, J., Richey, S., \& Freed, G. L. (2014). Effective Messages in Vaccine Promotion: A Randomized Trial. PEDIATRICS, 133(4), e835-e842. doi:10.1542/peds.2013-2365

Pfattheicher, S., Nockur, L., Böhm, R., Sassenrath, C., \& Petersen, M. B. (2020). The emotional path to action: Empathy promotes physical distancing and wearing of face masks during the COVID-19 pandemic. Psychological Science, 31(11), 1363-1373. doi: 10.1177/0956797620964422

Pfattheicher, S., Petersen, M. B., \& Böhm, R. (2021). Information about herd immunity through vaccination and empathy promote COVID-19 vaccination intentions. Health Psychology. Advance online publication. doi: $10.1037 /$ hea0001096

R Core Team (2020). R: A language and environment for statistical computing. Vienna, Austria: R Foundation for Statistical Computing. http://www.R-project.org/. [computer software].

Randall, D. M., \& Wolff, J. A. (1994). The time interval in the intention-behaviour relationship: Meta-analysis. British Journal of Social Psychology, 33(4), 405-418. doi:10.1111/j.2044-8309.1994.tb0

Robertson, E., Reeve, K. S., Niedzwiedz, C. L., Moore, J., Blake, M., Green, M., ... \& Benzeval, M. J. (2021). Predictors of COVID-19 vaccine hesitancy in the UK household longitudinal study. Brain, behavior, and immunity, 94, 41-50. doi: 10.1016/j.bbi.2021.03.008

Rosen, B., Waitzberg, R., Israeli, A., Hartal, M., \& Davidovitch, N. (2021). Addressing vaccine hesitancy and access barriers to achieve persistent progress in Israel's COVID-19 vaccination program. Israel journal of health policy research, 10(1), 1-20. doi: 10.1186/s13584-021-00481-x

Rosseel, Y. (2012). lavaan: An R Package for Structural Equation Modeling. Journal of Statistical Software, 48(2), 1-36. http://www.jstatsoft.org/v48/i02/. 


\section{VACCINATION: SELF-PERSUASION \& EMPATHY32}

Ruiz, J. B., \& Bell, R. A. (2021). Predictors of intention to vaccinate against COVID-19: Results of a nationwide survey. Vaccine, 39(7), 1080-1086. doi:10.1016/j.vaccine.2021.01.01

Schwarzinger, M., Watson, V., Arwidson, P., Alla, F., \& Luchini, S. (2021). COVID-19 vaccine hesitancy in a representative working-age population in France: a survey experiment based on vaccine characteristics. The Lancet Public Health, 6(4), e210-e221. doi:10.1016/s2468-2667(21)0001

Steiger, J. H. (2007). Understanding the limitations of global fit assessment in structural equation modeling. Personality and Individual Differences, 42(5), 893-898. doi:10.1016/j.paid.2006.09.017

Wilson, T. D., \& Brekke, N. (1994). Mental contamination and mental correction: Unwanted influences on judgments and evaluations. Psychological Bulletin, 116(1), 117-142. doi:10.1037/0033-2909.116.1.11

Yamey, G., Schäferhoff, M., Hatchett, R., Pate, M., Zhao, F., \& McDade, K. K. (2020). Ensuring global access to COVID-19 vaccines. The Lancet. doi:10.1016/s0140-6736(20)30763

Yıldırım, M., Geçer, E., \& Akgül, Ö. (2021). The impacts of vulnerability, perceived risk, and fear on preventive behaviours against COVID-19. Psychology, Health \& Medicine, 26(1), 35-43. doi: $\underline{10.1080 / 13548506.2020 .1776891}$ 